\title{
DESIGNING AND CONSTRUCTION OF THE SPECTRAL LIBRARY FOR TYPICAL PIGMENTS USED IN CHINESE PAINTINGS
}

\author{
S. Lyu ${ }^{1,2}$, Y. Liu ${ }^{1,2}$, M. Hou ${ }^{1,2, *}$ Z. Gao ${ }^{1,2}$, X. Yang ${ }^{1,2}$, M. Gu ${ }^{1,2}$ \\ ${ }^{1}$ School of Geomatics and Urban Spatial Informatics, Beijing University of Civil Engineering and Architecture, No.15 Yongyuan \\ Road, Daxing District, Beijing, China - lvshuqiang@bucea.edu.cn,2108521517004@stu.bucea.edu.cn, houmiaole@bucea.edu.cn, \\ gzh1314@163.com,15037089553@163.com,276683694@qq.com \\ ${ }^{2}$ Beijing Key Laboratory for Architectural Heritage Fine Reconstruction \& Health Monitoring, No.15 Yongyuan Road, Daxing
} District, Beijing, China

Commission II, WG II/8

KEY WORDS: Spectral Library, Pigments, Chinese Paintings, CH Documentation, Website

\begin{abstract}
:
Suffered from the environmental changes and human's influence, Chinese paintings are facing a worrying situation in documentation, which leads urgent into establishing digital archives to realize the permanent preservation of its surface information. Non-destructive identification of pigment types using hyperspectral techniques is the key to ensure the colour restoration scientifically. At present, one commonly used method for spectral identification is to calculate the similarities between unknown spectra and standard spectra in spectral library. The purpose of this study is to establish a spectral library of typical pigments specifically for the information preservation and pigment types identification on surface of Chinese paintings. The main works of the research are: (1) collecting the standard spectra in laboratory and the pigment spectra on surface of real cultural relics as the data source of the spectral library; (2) adding the expert knowledge of traditional pigments and normative descriptions of spectra measurement as part of the spectral library; and (3) constructing the spectral library website with the Browser/Server structure, and by using MySQL database for storage, SSM framework for background building and JSP page for displaying. The significance of the design and construction of the Typical Pigments of Chinese Paintings (TPCP) spectral library is not only to establish a standard spectral library for pigment types identification, but also a digital archive of pigment information on the surface of real cultural relics, to achieve permanent preservation, and provide spectral data sources and pigment information references for conservation workers and researchers in related fields for further research.
\end{abstract}

\section{INTRODUCTION}

Using modern technology to preserve information on surface of cultural relics to achieve the protection and inheritance of that is an important development trend in this field. Due to its finite surviving time and human damage, the colour on surface of painted artefacts such as murals and Chinese paintings will fade or lost to some extent. It requires the colour restoration, based on the digitally reserved pigments information, which exactly reflects the necessity and urgency of creating digital archive for cultural relics.

In order to restore the original colour of the cultural relics more scientifically, it is necessary to identify the type of pigment using non-destructive techniques before repair. In recent years, more and more researchers have used hyperspectral techniques to obtain pigment spectra, with non-contact, non-destructive, and more rapidly way, to achieve the identification of pigment types (Grabowski et al. 2018; Bai, 2002; Bioucas-Dias et al. 2008; Wang et al. 2015; Li et al. 2018; Vitorino et al. 2015; Daniel et al. 2016). At present, one commonly used method for spectral identification is to calculate the similarities between unknown spectra and standard spectra in spectral library. Currently, there are several international spectral libraries available (Wan, 2001), such as USGS-MIN (Hoefen et al. 2015), JPL, JHU (Pal et al. 2011), IGCP-264, and ASTER (Baldridge et al. 2009): (1) The USGS-MIN spectral library was established by the United States Geological Survey's Spectroscopy Laboratory, which contains spectra collected in laboratory, in field, and in aviation, and includes laboratory samples of specific minerals, plants, compounds, and man-made materials; (2) JPL spectral library was established by the Jet Propulsion Laboratory and tested 160 common minerals; (3) JHU spectral library was provided by Johns Hopkins University, including 15 sub-libraries; (4) IGCP264 was established by the United States for the project, using five spectrometers to test 26 samples and finally completed five spectral libraries; and (5) ASTER spectral library was established by the California Institute of Technology, with database search capabilities, and data sources from the USGS, JPL, and JHU spectral libraries. The above-mentioned several international spectral libraries have been widely applied in scientific research in the fields of geology and remote sensing. However, there are few spectral libraries established for the research hotspots of typical pigments on the surface of cultural relics.

In order to further promote the digital preservation and type identification of pigment spectra on surface of Chinese paintings, to achieve more complete information archiving and scientific colour restoration, based on studies on and experiments of laboratory samples and real cultural relics, the study designed and constructed a typical pigments spectral library- TPCP Spectral Library, which is specially for information preservation and type recognition, displaying on the website to share spectral data and pigments information.

In Section 1, the paper starts from an overall introduction of the necessity of the digital documentation and spectral library

\footnotetext{
* Corresponding author
} 
establishment for Chinese paintings. Section 2 describes the materials selection, the samples preparation, the instruments for data collection and chemical analysis, and the spectral acquisition process on surface of real cultural relics. The introduction of development techniques, development project tools, and website requirements analysis used in the design, construction, and management of the spectral library are contained in Section 3. Further, the interface of the spectral library and some of the module contents are displayed in Section 4. In the last section, the conclusion is briefly stated and future work is outlined as well.

\section{THE DATA SOURCS OF SPECTRAL LIBRARY}

\subsection{The Materials and Samples of Standard Spectra in Laboratory}

The standard spectra in TPCP spectral library are obtained based on laboratory-made pigment samples, including the no-glued solid pigments and the glued pigments are painted on the rice paper and simulated mural block. Both of them are performed data collection with ASD spectroradiometer.

The suitable pigments selected for spectral library establishment is critical for spectral identification. After careful market investigation and comparison, 32 kinds of commonly used pigments from a famous brand called Jiangsi Xutang were selected for samples making, of which 24 are mineral pigments and 8 are commonly used botanical pigments and chemical pigments. The no-glued solid pigments were placed in the cell plate for spectral collection (Figure 1 (a)), while the pigments, which had been thoroughly mixed with a certain concentration of glue, needed to be drawn on the rice paper and on simulated mural block before spectral collection. The following will further introduce the methods for sample making on two carriers.

2.1.1 Glue Selection and Preparation: There are many types of glue for painting, and the choice of them varies depending on the region. In the study, the glue produced by Jiang Sixutang was selected and used as following steps: firstly, to soak the desired amount of glue with 4 times cold water, and then to add 6 times boiling water at about $80{ }^{\circ} \mathrm{C}$ after it had expanded. It can be used until stirring for a while.

2.1.2 Design of paper pigment samples: In the study, the sample block of paper pigment was designed to be a size of 4 $\mathrm{cm} \times 4 \mathrm{~cm}$ to ensure a sufficient space for spectral data collection, and the brush full of pigment can fill it up just once (Figure 1 (b)). The Raw Xuan type of paper from Rongbaozhai, with no aluminum on surface, was selected for base material to reduce the influence on spectrum. Most samples were painted 1 layer, 2 layers, and 3 layers with a brush, respectively, to get three different filling thickness to ensure that the absorption characteristics of pigment components also can be reserved and observed for research as the thickness of the samples changed.

2.1.3 Design of simulated mural pigment samples: The base layer of mural painting was simulated by mixing loess with the appropriate amount of fine sand, with the approximate thickness of $2 \mathrm{~cm}$. Then, to brush lime water as the cover layer after drying, followed by drawing on its surface (Figure 1 (c)). The size of each simulated test block was $10 \mathrm{~cm} \times 10 \mathrm{~cm}$, and the painting area was $7 \mathrm{~cm} \times 7 \mathrm{~cm}$.

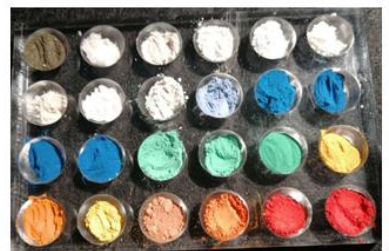

(a)

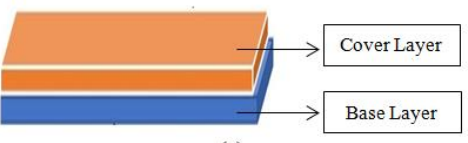

(c)

Figure 1. (a) some of no-glue powdered pigment samples;(b) some of paper pigment samples; and (c) some of simulated mural painting samples

\subsection{The Instruments for Spectral Data Collection and Chemical Analysis}

2.2.1 The Spectral Point Data Collection for Spectral library: The instrument used to acquire the spectral point data in this study was ASD FieldSpec4 portable spectroradiometer, with $350-2500 \mathrm{~nm}$ wavelength range, whose picture and specific parameters were shown in Figure 2. There are two ways to perform spectral acquisition using the ASD spectroradiometer: one is to use a well-sealed probe to collect spectra on the block area of the measurement object; the other one is to take out the optical fibre for spectral acquisition on smaller areas. Generally, the choice of the two perform ways depends on the size of the region of interest (ROI): for the measurement object with a large area of interest, the former is selected, and the halogen light source provided by the probe is used for illumination; for the measurement object with a small area of interest, in order to ensure that the spectra of the non-interest region would not be involved, the latter is selected, and an external halogen source is used for illumination. The data collection was carried out indoor to avoid the influence of other environmental factors. The halogen lamp, which can provide a source of light that is closest to sunlight, was selected as the artificial light source for spectral acquisition.

In this study, with a full consideration about the scope of the probe can be collected, the sizes both of the laboratory paper and the simulated mural pigment samples was designed to $4 \mathrm{~cm} \times 4 \mathrm{~cm}$ and $7 \mathrm{~cm} \times 7 \mathrm{~cm}$, respectively, which were larger than the block size that the probe can cover. Thus, the perform way for collecting standard spectra in laboratory was to use probe. While the perform way for spectral collection on surface of real cultural relics was to use optical fibre because the pigment mixture on the surface is complex and the area of the pure interest area is small. In the process of spectral acquisition, in order to reduce the influence of errors caused by light source and operation, the measurement was performed by rotating the probe or optical fibre along clockwise to four angles, respectively measuring four spectra, and finally averaging as the average spectra for each ROI. 


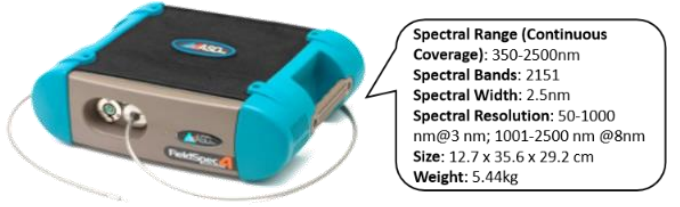

Figure 2. Picture and parameters of ASD FieldSpec4 portable spectroradiometer

2.2.2 The Instruments for Chemical Analysis of Pigments in Spectral Library: (1) Electron Microscope: KEYENCE VHX-600K ultra-depth-of-field three-dimensional microscope, whose maximum resolution approaches to the limit of optical microscope, can be used for observing the microscopic world that traditional optical microscope cannot be seen due to its insufficient depth of field. 3CCD\&Actuator, with real-time 2D \& $3 \mathrm{D}$ image connection, realizes 2D surface topography observation and 3D display in different depth of field for experimental samples. (2) Raman Spectrometer: Horiba Jobin Yvon XploRA laser confocal micro-Raman spectrometer, with three lasers of $785 \mathrm{~nm}, 638 \mathrm{~nm}$ and $532 \mathrm{~nm}$, automatic platform and Raman spectroscopy standard spectrum database software is equipped with the Olympus BX51 optical microscope that can observe and locate samples and is a combination of Raman spectrometer and optical microscope. Laser confocal microRaman spectrometer uses laser as the excitation source, which can be used for scanning point by point, line by line and face by side, and is equipped with three lasers that can be quickly switched to test different substances. According to the conjugate focusing principle, it has better imaging effects and higher resolution than traditional microscopes, and can analyse samples of different levels and depth of field. (3) Scanning Electron Microscope: JOEL JSM-6610LA scanning electron microscopeenergy spectrometer is divided into scanning electron microscope (SEM) part and X-ray energy spectrometer part. The SEM uses tungsten filament electron gun, including SEM secondary signal, backscatter and other imaging signals, the accelerating voltage is $0.3-30 \mathrm{KV}$, and the highest magnification can reach 300,000 times. And the EX-94300S4L1Q X-ray energy spectrometer can be used with electron microscope for microscopic morphology observation and elemental analysis of various materials. It can perform point analysis, line analysis and surface analysis of elements for different samples or different needs. (4) Portable X-ray Fluorescence Spectrometer: Thermo Fisher Scientific NITON XL3T900, portable X-ray Fluorescence Spectrometer (XRF), can analyse more than 40 elements, with a highperformance miniature X-ray tube, a measurement time of 60 seconds and a soil mode.

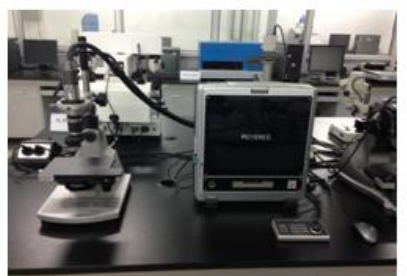

(a)

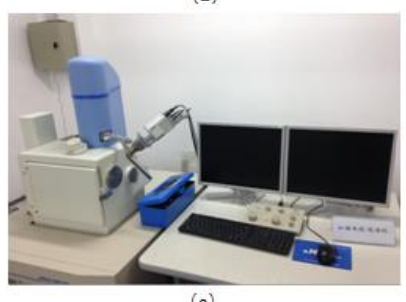

(c)
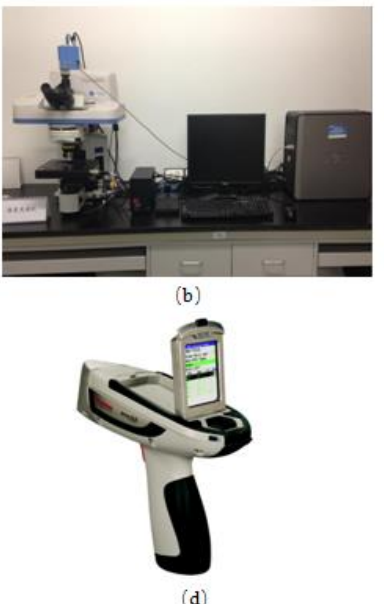

Figure 3. (a) electron microscope; (b) Raman spectrometer; (c) scanning electron microscope; and (d) portable X-ray fluorescence spectrometer

\subsection{The Spectral Collection on Surface of Real Cultural Relics}

In order to achieve the perpetual preservation of the pigment information of painted artefacts, the spectral data collected on the surface of real cultural relics is stored, managed, and displayed respectively in the TPCP spectral library according to the age and geography. Qutan Temple, which is included in TPCP spectral library, is located in Ledu County, Haidong City, Qinghai Province. The Tibetan Buddhist temple was built in 1392 and was famous for its precious cultural relics and the huge colourful mural paintings made by the court artists of the Ming and Qing Dynasties.

The spectral data of Qutan Temple mainly contains the Baoguang Hall, and the East and West Corridors of the temple. The instrument for obtaining the spectral point data is ASD spectroradiometer. Since the area of each mural painting, the maximum reaches $15.07 \times 5.18$ meters, is too large to completely collect the spectral point data of the entire painting. Therefore, the spectral acquisition can be focused on some ROIs according to different colours. In order to reduce the influence of subjective on the ROI selection, and to distribute these areas as evenly as possible over the entire mural surface, a grid can be drawn on the orthophoto of the mural painting firstly, each corner point of whose can be regarded as one ROI. Then, these ROIs can be classified according to different colours and analyzed respectively. Due to the randomness of the grid generation, in the line-by-line spectral acquisition process, if the pigment in the corner point has been shedded, faded, or mixed too complicated, it can be discarded and jumped to the next point. 


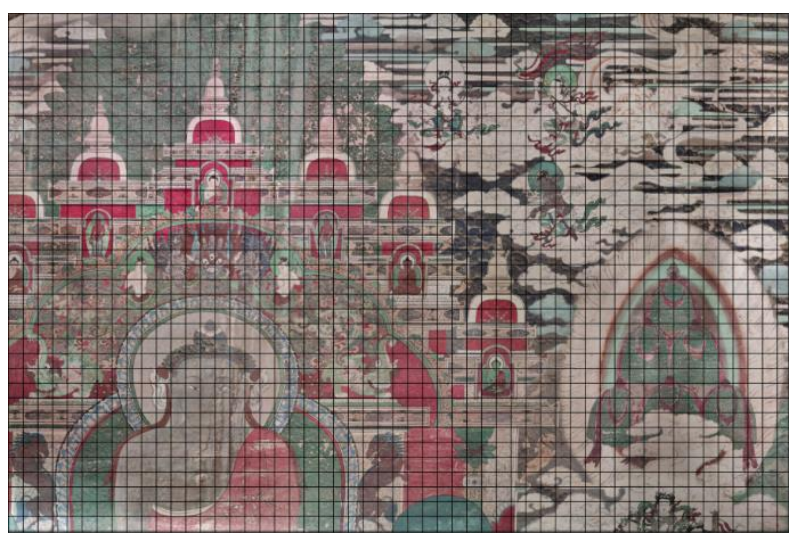

Figure 4. The grid generation on the mural painting of Qutan Temple

\section{THE CONSTRUCTION AND MANAGEMENT OF SPECTRAL LIBRARY}

\subsection{Related Development Technologies}

3.1.1 JSP: JSP (Java Server Pages), which has realized the extension for java language in HTML syntax, can be executed on the server side for using. It usually returns HTML text to the client, so the client just needs to browse the JSP interface under the browser. JSP has been widely used today, and almost all of Java-based web developments uses JSP as a front-end page for displaying (Liu, 2004).

3.1.2 SSM: The SSM is a framework, refers to Spring+SpringMVC+MyBatis (Cui et al. 2018), which realizes a whole set of logic: the request sent by page is handed to the controller for processing, and then the controller calls the processing logic of the business layer, the logical layer sends a request to the processing layer, and the persistence layer interacts data with the database, followed by returning the result back to the business layer, who then the business layer sends the processing logic back to the controller, and the controller invokes the view layer to present the data to the user for use.

\subsection{Development Tools}

This system is developed by Eclipse+MySQL+Tomcat7.0.

Eclipse is an open source, Java-based scalable development platform (Murphy et al. 2006). It can be used to greatly improve the efficiency of database and Java development, release, and integration of application-level program servers.

MySQL is a small relational database management system that is often used (Giacomo, 2005). Because of its light weight, low code complexity, and visual operations, MySQL has been widely used in many small and medium-sized website constructions in recent years.

The origin of Tomcat is the service provided by the Apache Software Foundation's JavaServlet container and Web server implementation. It is good at responding to user requests, and then gives users the application to present dynamic and static web pages, and finally sends dynamic and static data transmission results to user's browser (Gao et al. 2008).

\subsection{Requirement Analysis}

3.3.1 User-Role Requirement Analysis: Before constructing the spectral library, two types of user roles will under consideration, including general users and administrators. The authorities of the two are different: general users can search and use the data, while the administrators are capable add, delete, change, and check the data, and have the right to add an account. It promotes the determination of the entire spectral library business process (Figure 5), providing the logical support for subsequent spectral library development.

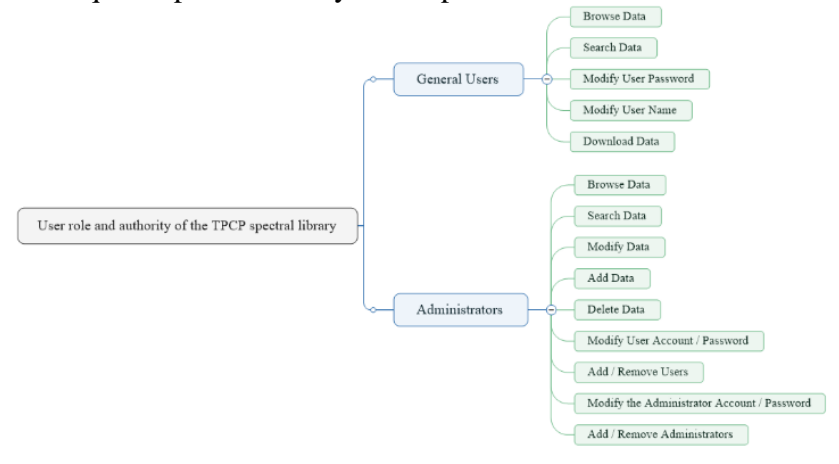

Figure 5. User role and authority

3.3.2 Performance Requirement Analysis: (1) For the user, the clear page function and obvious action buttons of the developed system are required, trying to ensure that system operators would not be limited by their computer level when using the spectral library, and the system response time would be less than 10 seconds; (2) For the administrator, the simple and convenient operation interface are required the make the database maintenance easily; and (3) The spectral library needs to be relatively stable, and the administrator should check it regularly.

3.3.3 Overall business framework of spectral library: The TPCP spectral library system uses a browser as the main client. The user layer includes general users and administrators, all of which enter the relevant pages of the system by the browser operation. The different operation requests from user are submitted to the server through the network, which would then perform interactions to the requested data by using implementation language of the database and the logic layer, followed by returning the data processing results by the network back to users for use (Figure 6).

The business framework of spectral library contains three parts: the front end, the back end, and the database. The spectral library is connected by JDBC, and is guided by the business flow to enter the application layer for logical processing. The data, which has been processed by the application layer, will be uploaded to the web end in the background, which is going to process the data for another time. It is a logical process of receiving the background data and then classified displaying on the browser. The spectral library is written in the JavaScript language and then delivered for the user's browser via the network, which can be accessed and operated by users.

The network request uses the HTTP, which refers to the transport protocol used by the Internet server to transmit hypertext to the local browser. HTTP is a method for passing data based on TCP/IP communication protocol (whose data is usually consisted of HTML files, image files, query results, etc.). 


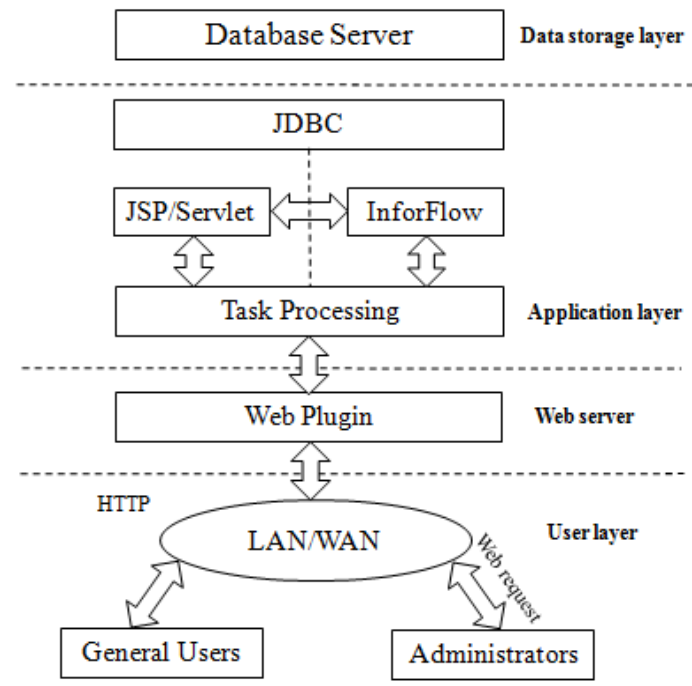

Figure 6. Overall business framework of TPCP spectral library

\section{SPECTRAL LIBRARY INTERFACE AND MODULE DISPLAY}

The home page provides a brief description of the TPCP spectral library and introduces the main contents of four modules, including the "Laboratory Standard Pigment Spectra", the "Pigment Spectra on Surface of Real Cultural Relics", the "Expert Knowledge", and the "Normative Description". The overall interface is simple and the navigation is clear and intuitive (Figure 7 and Figure 8). The contents of the first two modules have been described in detail in section 2 , and the following will briefly introduce the contents of the latter two modules.

\subsection{The Expert Knowledge}

In addition to the spectral data of the pigments, the spectral library also contains relevant expert knowledge of pigments commonly used in ancient time of China. The spectral absorption positions of the main ions contained in the pigments are summarized by combining the related work reviews with the standard pigment spectra curves. The main cations include $\mathrm{Cu}^{2+}$, $\mathrm{Fe}^{2+}, \mathrm{Fe}^{3+}, \mathrm{Hg}^{+}$, whose absorption positions generally appear before the wavelength of $1000 \mathrm{~nm}$. The main functional groups are $\mathrm{CO}_{3}{ }^{2-}$ and $\mathrm{OH}^{-}$, whose absorption positions generally appear after the wavelength of $1000 \mathrm{~nm}$

\subsection{The Normative Description}

According to the military standard of China "Code for measurement of spectra (GJB-4029-2000)" and other standards, this study standardizes the spectral data acquisition process for the spectral library, which provides a basis for the scientific storage and systematic management of pigment spectra.

In addition to the spectral curves from the visible to near-infrared range acquired by hyperspectral instruments, the library also provides a detailed description of each pigment, including the reserved form, chemical formula, and the analysis results by using electron microscopy, Raman spectroscopy, and SEM. And the purity and composition of the pigment samples are verified using the results of the X-ray fluorescence spectrometer. Taking mineral pigment Malachite as an example, the description using various chemical analysis means is partially shown in Figure 9 and Table 1.

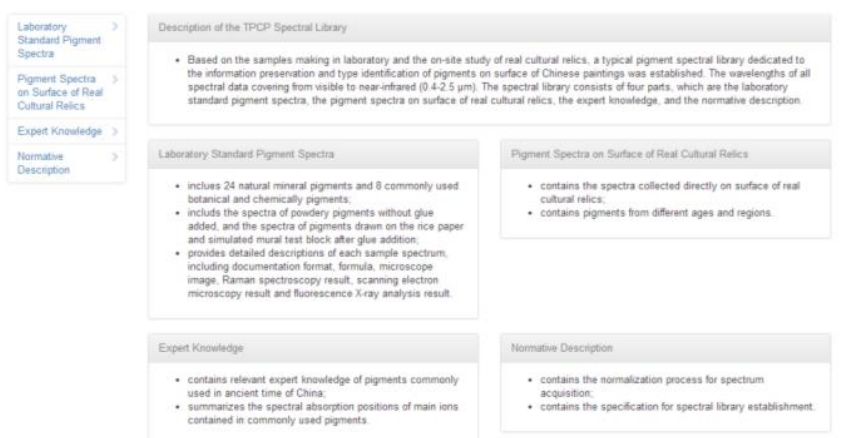

Figure 7. The home page of TPCP spectral library

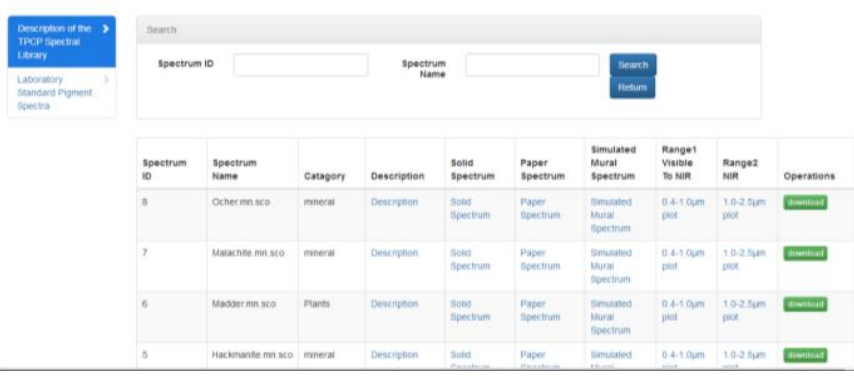

Figure 8 . The module interface of TPCP spectral library

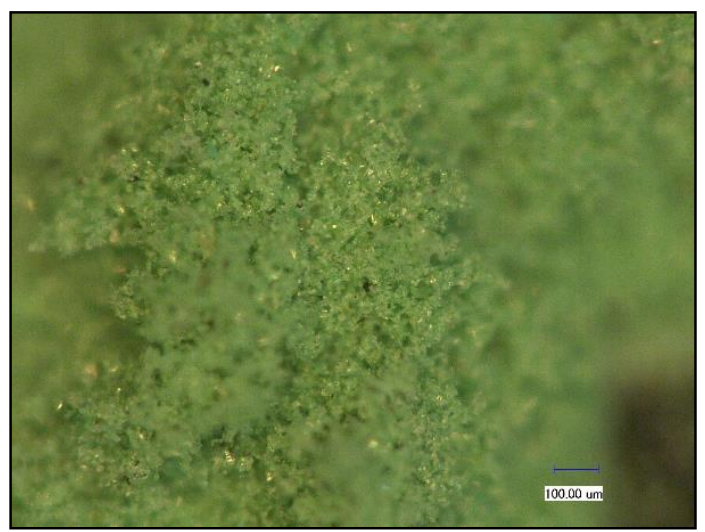

(a)

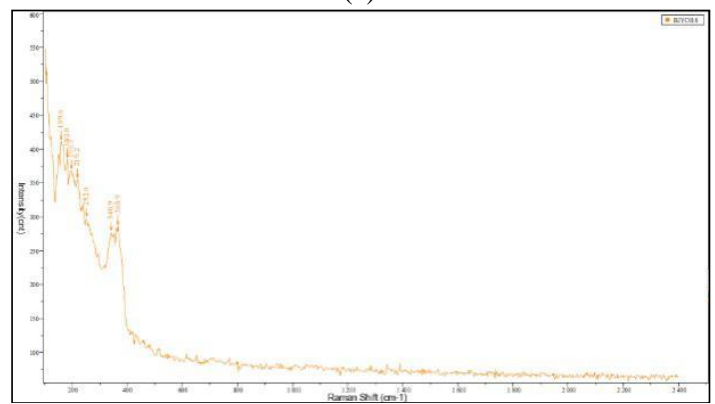

(b) 


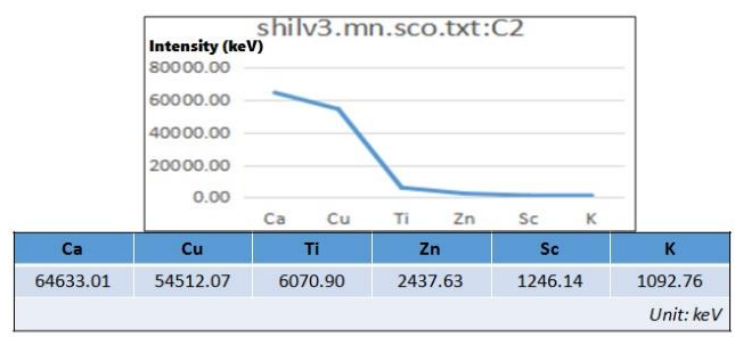

(c)

Figure 9. (a) electron microscope result; (b) Raman spectrometer result; and (c) portable X-ray fluorescence spectrometer result

\begin{tabular}{|l|c|c|c|c|}
\hline Element & $(\mathrm{keV})$ & Mass\% & Error\% & Atom\% \\
\hline $\mathrm{Cu}$ & 8.040 & 48.32 & 0.74 & 17.80 \\
$\mathrm{O}$ & 0.525 & 37.22 & 0.20 & 54.47 \\
$\mathrm{C}$ & 0.277 & 14.05 & 0.28 & 27.38 \\
$\mathrm{Al}$ & 1.486 & 0.40 & 0.17 & 0.35 \\
Total & & 100.00 & & 100.00 \\
\hline
\end{tabular}

\section{CONCLUSIONS}

The design and construction of the TPCP spectral library aims to realize the function of digital documentation of pigments information and pigment types identification on the surface of painted artefacts. Faced with the urgent need for digital documentation of cultural relics, this study establishes digital archives for painted artefacts, such as the mural painting of Qutan Temple, which carried Tibetan Buddhism culture and was built in the Ming and Qing Dynasties. These archives, including pigment information, age information, and region information, are systematically managed in the TPCP spectral library, and displayed on website, for sharing to more researchers in related fields to trace history and study regional culture by referring to the material selection and painting style on surface of these real cultural relics. Faced with the common problem of the lack of scientific basis in colour restoration of painted artefacts, this study uses different base materials to carry different pigments to simulate the varies formats of painted artefacts as more as possible, followed by collecting the spectra on surface of these laboratory-made samples as the laboratory standard spectra, which is the support for pigments identification by using the spectral library.

The TPCP spectral library is still in the process of continuous improvement: (1) the number of samples, the types of pigment, and the forms of carrier will be expanded to make the standard spectra contained in the spectral library more complete; (2) with the accumulation of team projects, digital archives will be created for more painted artefacts; and (3) the data preserved in onedimensional form at present will also be developed to twodimensional or even multi-dimensional data archiving, to realize more completely digital documentation for pigment information, historical and human memory on the surface of cultural relics.

\section{ACKNOWLEDGEMENTS}

The authors would like to acknowledge the Conservation Institute of Dunhuang Academy for its support of this work, and thank the professor, Songnian $\mathrm{Li}$, who has offered many precious suggestions in academic study.
This work was supported by the Research Fund of the National Key Research and Development Program (No. 2017YFB1402105), the Fundamental Research Funds for Beijing University of Civil Engineering and Architecture (No. X18024), the Scientific Research Program of Beijing Education Commission (KM201010016002), and the Importation and Development of High-Caliber Talents Project of Beijing Municipal Institutions (21147515209).

\section{REFERENCES}

Baldridge, A. M., Hook, S. J., Grove, C. I., Rivera, G., 2009. The aster spectral library version 2.0. Remote Sensing of Environment, 113(4), pp. 711-715.

Bai, J. W., 2002. The study on the spectral mapping technique based on the hyperspectral database. Doctoral dissertation, Institute of Remote Sensing Applications Chinese Academy of Sciences, China.

Bioucas-Dias, J. M., Nascimento, J. M. P., 2008. Hyperspectral subspace identification. IEEE Transactions on Geoscience and Remote Sensing, 46(8), pp. 2435-2445.

Cui, Y., Jiang, L., 2018. Design of batch audio/video conversion platform based on javaee. In: IOP Conference Series, Materials Science and Engineering, Vol. 322, No. 6.

Daniel, F., Mounier, A., Pérez-Arantegui, J., Pardos, C., PrietoTaboada, N., de Vallejuelo, S. F. O., Castro, K., 2016. Hyperspectral imaging applied to the analysis of goya paintings in the museum of zaragoza (spain). Microchemical Journal, 126, pp. 113-120.

Gao, Z., Kang, X. J., 2008. Research on enhancing the running performance of tomcat server. Computer \& Digital Engineering, 10.

Giacomo, D. M., 2005. Mysql: lessons learned on a digital library. IEEE Software, 22(3), pp. 10-13.

Grabowski, B., Masarczyk, W., Glomb, P., Mendys, A., 2018. Automatic pigment identification from hyperspectral data. Journal of Cultural Heritage, 31, pp. 1-12.

Hoefen, T. M., Kokaly, R. F., Swayze, G. A., Livo, K. E., 2015. Evaluating field spectrometer performance with transmission standards: examples from the usgs spectral library and research databases. In: AGU Fall Meeting Abstracts.

Li, J. F., Wan, X. X., 2018. Non-destructive identification of mineral pigments in ancient murals by visible spectroscopy. Spectroscopy and Spectral Analysis, 38(1), pp. 200-204.

Liu, C. H., 2004. Data flow analysis and testing of java server pages. In: Proceedings of the 28th Annual International Computer Software and Applications Conference, Vol. 2, pp. 114-119.

Murphy, G. C., Kersten, M., Findlater, L., 2006. How are java software developers using the elipse ide? IEEE Software, 23(4), pp. 76-83.

Pal, S. K., Majumdar, T. J., Bhattacharya, A. K., Bhattacharyya, R., 2011. Utilization of landsat $\mathrm{ETM}+$ data for mineraloccurrences mapping over Dalma and Dhanjori, Jharkhand, India: 
an advanced spectral analysis approach. International journal of remote sensing, 32(14), pp. 4023-4040.

Vitorino, T., Casini, A., Cucci, C., Melo, M. J., Picollo, M., Stefani, L., 2015. Non-invasive identification of traditional red lake pigments in fourteenth to sixteenth centuries paintings through the use of hyperspectral imaging technique. Applied Physics A, 121(3), pp. 891-901.

Wan, Y. Q., 2001. The analysis of mineral and rock's hyperspectral library. Geo-information Science, 3(3), pp. 54-58.

Wang, L. L., Li, Z. M., Ma, Q. L., Mei, J. J., 2015. Nondestructive and in-situ identification of pigments in wall painting using hyperspectral technology. Dunhuang Research, pp. 122128. 